\title{
Research on the Conflict Analysis of Supply Chain Management based on Graph Model with MRCR
}

\author{
Hongzhuan Chen \\ College of Economics and Management \\ Nanjing University of Aeronautics and Astronautics, \\ Jiangsu Province, China \\ 13813922476@163.com
}

\section{Lulu Song}

College of Economics and Management

Nanjing University of Aeronautics and Astronautics, Jiangsu Province, China 562858711@qq.com

\author{
Xuesong Zhuang \\ College of Economics and Management \\ Nanjing University of Aeronautics and Astronautics, \\ Jiangsu Province, China \\ 136236312@qq.com \\ Ting $\mathrm{Li}$ \\ College of Economics and Management \\ Nanjing University of Aeronautics and Astronautics, \\ Jiangsu Province, China \\ 562858711@qq.com
}

\begin{abstract}
In this research, the conflict problem in supply chain management is studied. The method of graph model is applied to solve the conflict problem in supply chain management, then based on the matrix form, the decision support system(MRCR) is used to make auxiliary decision, considering the uncertainty to select the strategy of decision makers in supply chain management. And an empirical analysis about the conflict problem is taken in YJ Nanjing Company's supply chain management. On the basis of the characteristics of YJ Company supply chain management, model is established, then, equilibrium point is obtained by MRCR, finally, make a decision to resolve the conflict after analysis.
\end{abstract} MRCR

Keywords- supply chain management, conflict, graph model,

\section{INTRODUCTION}

Supply chain management refers to using system management, operations research, management science, decision support systems, information technology and other science managing methods to plan, coordinate and control the information flow, logistics, capital flow, value flow in the supply chain. Owing to the differences in awareness and target, the providers often give priority to the realization of their own goals, then, severe conflict of supply chain management will happen when the external environment and internal resources of supply chain change.

Many authors studied the conflict problems in supply chain management. Based on the perspective of the causes of conflict, Jiling $\mathrm{Hu}$, Qing Fang proposed that supply chain management conflict is "the inconsistent or opposite to each other in target, interests and awareness among at least two supply chain node enterprises, thus leading to internal or external conflict, then further confrontation or clashes" [1] White oak Phil proposed that errors of forecasting and delivery has caused conflicts among supply chain members, and the bigger orders, the longer replenishment cycle, the more probability an error will occur, the more conflicts between manufacturers and retailers ${ }^{[2]}$. Ravindra Krovi believed that information viscous reflects the conflict degree of supply chain nodes, uncertainties associated with information viscous eventually led to the bull whip effect, which material piled up along the supply chain, leading to excess inventory ${ }^{[3]}$. Xuesha nusing the method of graph model to make an empirical analysis of the conflict of technology transfer in the process of complex product development, calculated the equilibrium of the conflict, and provided the simulation of negotiation and consultation process between policy makers ${ }^{4]}$. Song Hua found nonmandatory power and trust benefit conflict resolution, promote cooperation between supply chain members and the development of supply chain alliance ${ }^{[5]}$. Zhao wei introduced the graphic model of conflict analysis, analyzed actual situation and conflict equilibrium, focused on corresponding shortest path to achieve equilibrium ${ }^{[6]}$. Kevin and Hipel divided the four basic equilibrium solution definition of graph model into a, b, c, d four kinds of circumstances, conflict analysis problem is fully discussed under the incomplete information and uncertain preference ${ }^{[7]}$. Hamouda established a new three-level preference strength structure weak (better than, stronger than, weak strong than) instead of the original simple preference, and introduces three preferences in stability study, discuss the impact that the preference strength made on the equilibrium of the model. [8].

\section{TYPE STYLE AND FONTS}

In supply chain management, there are many kinds of conflicts. Based on the impact, content and scope, the conflict of supply chain management can be classified. Firstly, it can be divided into constructive conflict and destructive conflict according to the conflict effects of supply chain conflict. Secondly, it can be divided into goal conflict, cognitive conflict, emotional conflict and procedures conflict according to the content of the conflict. Then, it can be divided into each enterprise internal conflicts, the same node conflict between enterprises and adjacent nodes conflict between enterprises in the supply chain. And there are four 
main types of conflict in supply chain with manufacturers as the core, including: conflict between suppliers and suppliers; conflict between the supplier s and the manufacturer; conflict between dealers and dealers; conflict between dealers and manufacturers.

\section{CONFLICT ANALYSIS GRAPH MODEL}

\section{A. Graph Model}

Conflict analysis graph model is represented by $\mathrm{V}=\{\mathrm{N}, \mathrm{S}$, $\mathrm{P}, \mathrm{G}$ \}. Among, $\mathrm{N}(\mathrm{N} \geq 2)$ represents non-empty collection of all decision makers, each decision makers choose a strategy, and all strategy combinations form a state; S represents nonempty collection of all decision makers; P represents the preference information of decision makers; $G$ represents state transition diagram. Apply graph model to solve the conflict, firstly, understand and describe reality conflict; secondly, abstract the reality of conflict as a mathematical model; then, seek a balanced solution of conflict after modeling and stability analysis of process. Modeling process includes identifying the conflict decision makers and strategies, analysis of possible States and state transitions and preferences information of decision makers. Stability analysis is mainly calculate individual stability and global stability $^{[9]}$.

If there are $\mathrm{N}$ decision makers and $\mathrm{S}$ possible states in a graph model, the preference of decision makers $i$ is encoded by preference relation $\{>i, \sim i\}$ of state $S$ (such as, state $s, q)$. "s $>$ iq" represents decision makers i prefers state s; "s $\sim$ iq" represents decision makers $i$ has the same preference to the two states. Generally, to each decision makers i, we assume that $>\mathrm{i}$ is irreversible, $\sim \mathrm{i}$ is reversible, but when the preference information is very difficult to obtain or not sure, there would cause problems, for example, bring about uncertainty preferences. So a new preference structure is proposed by some scholars, which is $\{>\mathrm{i}, \sim \mathrm{i}, \mathrm{Ui}\}$, the meaning of $>\mathrm{I}$ and $\sim \mathrm{I}$ is consistent with simple preference structure, "sUiq" represents decision makers i might tend to state $\mathrm{s}$, also might tend to state q. sUiq reflects that decision makers $i$ is lack of the preference information of state $s, q$, or decision makers i don't want to make a comparison between two states. Preference uncertainty theory expands the application range of the existing method of graph mode ${ }^{[10]}$. Currently, MRCR system has realized the uncertain preference input, which makes the conflict decision analysis system more accurate on the analysis of the case model.

\section{B. Stability Analysis in Graph Model}

The main problem of the stability analysis is: whether a decision maker would choose to removed from one state where possible or not. So a steady state is that decision makers will not urge unilaterally to remove from this state. If all the decision makers achieve stability in a certain state point, the state is stable for all decision makers, and says the point is an equilibrium point. It is one solutions of the decisions conflict analysis.

According to the different decision-making style and background, there are four kinds of concepts about the solutions in the stability analysis (i.e. stability definition), including: Nash,GMR,SMR and SEQ ${ }^{[11]}$. GMR equilibrium includes the GMR、SEQ and Nash equilibrium. It shows that the stability of the Nash is the best, and the stability of the GMR is the weakest. In this paper, the stability analysis is mainly used under uncertain preference information. Based on uncertain preference structure, uncertain stability divides into the new four kinds of stability under four kinds of basic stability. With a, b, c, d on behalf of the four new form of stability definition, each basic stability can be divided into four kinds of circumstances, a total of 16 kinds.

\section{APPLICATION CASES}

\section{A. YJ Nanjing Company Profiles}

In this case, YJ Nanjing Company supply chain management conflict is taken as a example. YJ Nanjing is a Company which produce car seats taken by JIT, it supplies for the major Nanjing local automotive manufacturers, Shanghai Volkswagen Company in Nanjing as one of the Company's major customers. Currently, the large suppliers of YJ companies mostly have established short warehouses in Nanjing in order to meet with JIT production requirements. This reduces both YJ Company site inventory, save inventory space and also improved controllability of the entire supply chain, reducing the out of stock due to incidents during transport.

Leather seating surfaces of YJ Nanjing Company are supplied by the Guoli Company, as Guoli set up no warehouse in Nanjing, in order to meet customer's supply, YJ had to set up more safety stocks, however, with Shanghai Volkswagen increasing production, YJ has been unable to provide so many venues. Every morning two cars arrive, it led to a lot of backlog, and leather jacket is often placed outside, products with damp or wet, causing difficulties in production. YJ presented that Guoli should set up warehouse in Nanjing, for cost reasons, Guoli have not yet replied. Because Guoli is specified sentinel providers, YJ hopes Shanghai Volkswagen can coordinated. SVW absolutely hope that YJ Company will meet its JIT production requirements, but if the two parties can coordinate by themselves and it will not want to intervene. This causes conflict.

\section{B. Conflict graph model of supply chain management for YJ Nanjing}

a) Decision Makers and Behaviors.

The YJ Nanjing Company's supply chain, there are three main decision makers: YJ Nanjing Company (DM1) 、 Guoli Company ( DM2 ) and Shanghai Volkswagen Company in Nanjing (DM3). Guoli as a sentinel providers of Shanghai Volkswagen, YJ Company cannot terminate cooperation; If Shanghai Volkswagen stops production as YJ Company failed to timely supply, YJ Company needs to bear the losses. Decision makers could take the three strategies as follows:

YJ Nanjing Company (DM1 ) : (1) Guoli set up short barge warehouse in Nanjing, and bear the relevant expenses; 
(2) Guoli set up short barge warehouse in Nanjing, costs borne by the two companies of YJ and Guoli.

Guoli Company (DM2): (1) Agreed to set up a short barge warehouse in Nanjing, bear the full cost;(2)Agreed to set up a short barge warehouse in Nanjing, bear the full cost with YJ;(3)Continue to delay time: Need to bear losses.

Shanghai Volkswagen Company in Nanjing (DM3): (1) Negotiations with Guoli and promote the establishment of the warehouse, make the mass production is not affected;(2) Leave them solve itself.

They are based on its own interests:

If YJ Company in Nanjing is unable to keep up with the mass production, lead to stop line, it will be required to pay damages of $\$ 7000$ per minute. For not affect production, requirements to set up warehouse in Nanjing will not be changed.

Guoli Company: do not want to pay all the costs generated by short warehouses, while the losses could be huge problems. Hope YJ Company may be granted on the establishment and management of warehouse.

Shanghai Volkswagen Company in Nanjing: hope that the two parties can resolve the issue, the most important thing is not to affect their production.

b) States and States Transfer

YJ has two choices, Guoli has three, and Shanghai Volkswagen Company in Nanjing has two. For their own interests, when YJ chooses action 2 "Guoli set up short barge warehouse in Nanjing, costs borne by the two companies of YJ and Guoli", the Shanghai companies must not be selected 1 "Agreed to set up a short barge warehouse in Nanjing, bear the full cost". According to the all possible states, form (Table 1) is as follows:

TABLE I. AVAILABLE STATES

\begin{tabular}{|c|l|l|l|l|l|l|l|l|l|l|l|}
\hline DM & $\boldsymbol{a c}$ & $\boldsymbol{S 1}$ & $\boldsymbol{S} 2$ & $\boldsymbol{S 3}$ & $\boldsymbol{S 4}$ & $\boldsymbol{S 5}$ & $\boldsymbol{S 6}$ & $\boldsymbol{S 7}$ & $\boldsymbol{S 8}$ & $\boldsymbol{S 9}$ & $\boldsymbol{S 1 0}$ \\
\hline \multirow{2}{*}{ DM1 } & 1 & 1 & 1 & 1 & 1 & 1 & 1 & 0 & 0 & 0 & 0 \\
\cline { 2 - 12 } & 2 & 0 & 0 & 0 & 0 & 0 & 0 & 1 & 1 & 1 & 1 \\
\hline \multirow{4}{*}{ DM2 } & 1 & 1 & 1 & 0 & 0 & 0 & 0 & 0 & 0 & 0 & 0 \\
\cline { 2 - 12 } & 2 & 0 & 0 & 1 & 1 & 0 & 0 & 1 & 1 & 0 & 0 \\
\cline { 2 - 11 } & 3 & 0 & 0 & 0 & 0 & 1 & 1 & 0 & 0 & 1 & 1 \\
\hline \multirow{2}{*}{ DM3 } & 1 & 1 & 0 & 0 & 0 & 1 & 0 & 1 & 0 & 1 & 0 \\
\cline { 2 - 11 } & 2 & 0 & 1 & 1 & 1 & 0 & 1 & 0 & 1 & 0 & 1 \\
\hline
\end{tabular}

Originally $2 * 3 * 2=12$ state, excluding the two combinations: the YJ choice behavior of 2, Guoli choice behavior of 1 , it is concluded that the above table of 10 different feasible conditions. state 1 means YJ and the Shanghai Company choose to set up short warehouse in Nanjing, fees paid by Guoli, Shanghai Volkswagen to promote conflict resolution. According to table 3.1 can conclude the state transition matrix ( $\mathrm{Ji}$ refers to state transition matrix of DMi):

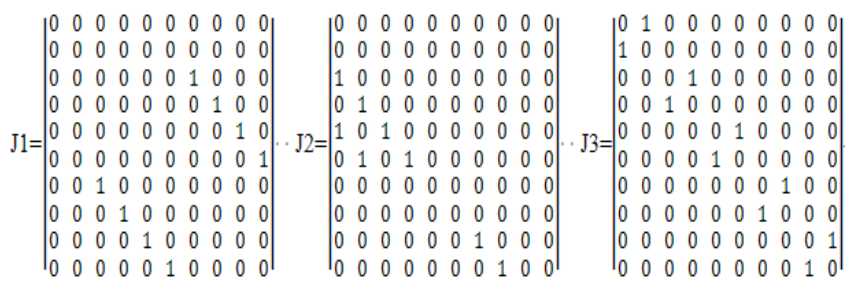

\section{c) Preferences and Graph Model}

For DM1, it hopes that related fees shall be borne by the DM2, most do not want the DM2 to chose delay strategy, so the DM1 prefer 1, 2 more than 3, 4, 7, 8, and 3, 4, 7, 8 more than 5,6,9,10. At the same time, the DM1 surely hopes DM3 can take positive measures to solve the conflict, promote the establishment of the warehouse. Therefore, DM3 preference information is as follows:

DM1: $\mathrm{S} 1>\mathrm{S} 2>\mathrm{S} 3>\mathrm{S} 4>\mathrm{S} 7>\mathrm{S} 8>\mathrm{S} 5>\mathrm{S} 6>\mathrm{S} 9>\mathrm{S} 10$

For DM2, its preference is uncertain, from long term and short-term interests, there are different preferences.

For long-term interest considerations, DM2 will agree to establish short warehouses to promote supply chain management. DM2 preferences for three policies: $2>1>3$. DM2 also hope the DM1 share the costs of the decision, and the DM3 could advance the settlement of the conflict. Therefore, under the long-term benefits, DM2 preference information is as follows:

DM2: $\mathrm{S} 7>\mathrm{S} 8>\mathrm{S} 3>\mathrm{S} 4>\mathrm{S} 1>\mathrm{S} 2>\mathrm{S} 9>\mathrm{S} 10>\mathrm{S} 5>\mathrm{S} 6$

For short term-interest considerations, DM2 does not agree to the establishment of short warehouse, it will give priority to adopt delaying tactics, followed by wish to bear the cost as little as possible. DM2 preferences for three policies: $3>2>1$. Therefore, under the short term interests, DM2 preference information is as follows:DM2: $\mathrm{S} 9>\mathrm{S} 10>\mathrm{S} 5>\mathrm{S} 6>\mathrm{S} 7>\mathrm{S} 8>\mathrm{S} 3>\mathrm{S} 4>\mathrm{S} 1>\mathrm{S} 2$

To sum up, DM2 uncertain information is as follows:

DM2: $\mathrm{S} 7>\mathrm{S} 8>\mathrm{S} 3>\mathrm{S} 4>\mathrm{S} 1>\mathrm{S} 2 ; \mathrm{S} 9>\mathrm{S} 10>\mathrm{S} 5>\mathrm{S} 6$

For DM3, it more hope do nothing, and the two sides could agreed to establish Nanjing warehouse, ensure the normal production, but as a threat to its own interests, it will also take the appropriate action. If DM1 and DM2 can build warehouse, both of which bear the relevant expenses by either party, DM3 will be satisfied, as a result, DM3 preference is divided into two parts, one contains the preference of uncertainty, and the other part is a simple preference:

$\mathrm{DM} 3$ uncertain preference information: $\mathrm{S} 8>\mathrm{S} 7 ; \mathrm{S} 2>\mathrm{S} 1$.

$\mathrm{DM} 3$ simple preference: $\mathrm{S} 4>\mathrm{S} 3>\mathrm{S} 10>\mathrm{S} 9>\mathrm{S} 6>\mathrm{S} 5$

Although DM3 separate the two pieces of information to consider, but DM3 of S8, S7, S2, S1, preference is always greater than S4, S3, S10, S9, S6 and S5. DM3 preference to the latter point is fixed.

According to the state transition matrix of three decision makers J1, J2, J3 can get state transition diagram model (Figure1) as follows: 


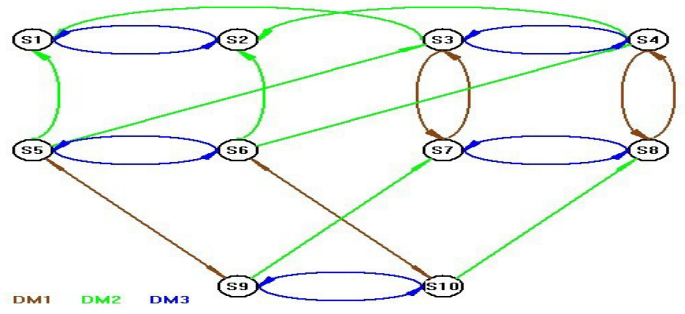

Figure 1. graph model

Brown arrows in Figure1 represent the DM1 transfer situation, green arrow is the transfer of DM2, and blue arrow is the transfer of DM3. There are both two-way transfer and one-way transfer between state points, such as S5 and S1 is a one-way transfer, can only be transferred from S5 to S1, cannot be transferred from S1 to S5. S5 and S6 is two-way transfer, can be transferred from S5 to S6, can also be transferred from S6 to S5.

\section{Stability Analysis Based on Decision Support System}

In order to obtain stable results, we use MRCR system to analyze the data. Firstly, input the decision makers information, there are three decision makers in this case conflict, acts to be chosen are 2, 3, and 2, then input feasible state points, according to state transfer matrix $\mathrm{J} 1, \mathrm{~J} 2$, and $\mathrm{J} 3$,input three decision makers of state transfer information, due to uncertainty preferences exist in YJ Company supply chain conflict, DM3 is special, so, firstly input simple preferences of DM3, then to input DM2 and DM3 uncertainty preferences information. Thus, we can get the stability result by MRCR system analysis in the case that there are uncertain preference between DM2 and DM3 .As shown in Figure2:

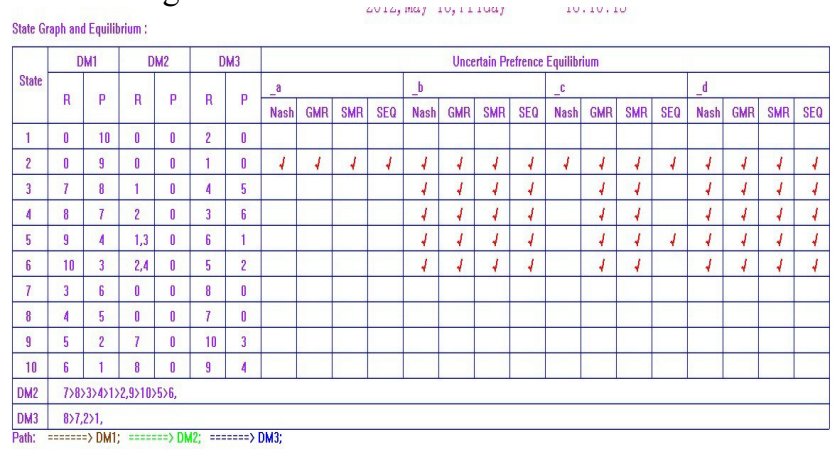

Figure 2. the stability results of uncertainty

From the above results, only state point 2 meets all kinds of balance requirements, and state $3,4,5,6$, partly meets the balance, effect is less than state point 2 . In state point 2, Nanjing YJ and the Guli set up short barge warehouse, and the expenses shall be borne by Guoli, Shanghai Volkswagen has not stepped in. This is obviously a good results for YJ which is in the middle reaches of the supply chain, this choice can be a complete resolution to conflict 3, 4, 5, 6, which shows that YJ Company hopes the fees shall be borne by Guoli, but the choice shared cost or delay time is based on their own interests. To share the benefit is under the long-term interests, delay time results of short-term interests. From this aspect it can be seen that balance of point 2 is the best.

\section{RESUlts}

In view of the stability results, the relevant decision recommendations are put forward:

Firstly, In order to make the supply chain to achieve the best balance of the conflict, the Guoli should try to choose "Agreed to set up a short barge warehouse in Nanjing, bear the full cost". It is helpful for future cooperation with YJ, but also to the whole supply chain management. Although preliminary paid a high cost, it worthwhile in the long-term interests.

Secondly, YJ should try to communicate with Guoli, make the decision to develop towards a favorable direction, adhere to the choice, to put pressure on the Company.

Thirdly, As YJ is a JIT supplier of Shanghai Volkswagen, for Shanghai Volkswagen, its own production requirement, in order to make the production line will not be shut down because of out of stock, it will support that establish a short barge warehouse in Nanjing, and easily reached an agreement with YJ Company in this point of view. So YJ Nanjing hope that Guoli can establish short barge warehouse and bear the full cost, with the support of Shanghai Volkswagen, it can put pressure on Guoli to promote the progress of the warehouse.

\section{ACKNOWLEDGMENTS}

The relevant researches done in this paper are supported by Natural Science Foundation of China (No. 71002046, No. 71372080 ); The Fundamental Research Funds for the Central Universities (No.NR2013017).

\section{REFERENCES}

[1] Jiling Hu, Liwei Yang.: Supply chain enterprise conflict studies [J] Scientific and technological progress and countermeasures, 2004,9:97-99.

[2] Hennart J.F.. A transaction costs theory of equity joint ventures[J]. Strategic Management Journal, 2006, 9(4): 361-374

[3] Ravindra Krovi, Akhilesh Chandra, Balaji Rajagopalan 2003: Information flow parameters for managing organizational process $[\mathrm{J}]$. Association for Computing Machinery. Communications of the ACM, Vo1.46, Iss. 2, pg. 77

[4] Xueshan Han, Haiyan Xu. Conflict Analysis of Technology Transfer Based on Graph Model [J]. Science \& Technology and Economy, 2012. 149: 72-76

[5] Hua Song. Research on the Impact of Power, trust to conflict resolution mechanism and its partnership $[\mathrm{J}]$. journal of management, 2009,11: 1437-1442.

[6] Wei Zhao. A conflict analysis method study Based on Graph Model [J]. Science and Technology Management Research,2010.20:231233

[7] Kevin W, Hipel. Preference Uncertainty in the Graph Model for Conflict Resolution[J]. IEEE Transactions on systems, man, and cybernetics, 2004,4(34):507-520.

[8] Hamouda, Kilgour, Hipel. Strength of preference in graph models for multiple decision maker conflicts[J]. Applied Mathematics and computation, 2006,179(1):324-327. 
[9] Haiyan Xu, Kevin W. Li,K. W. Hipel, and D. M. Kilgour: A Matrix Approach to Status Quo Analysis in the Graph Model for Conflict Resolution [J], Applied Mathematics and Computation. 2009, 212 (2):470-480.

[10] Hipel,Keith W,D.Marc Kilgour,Liping Fang,and Xiaoyong (John) Peng. The Decision Support System GMCR in Environmental
Conflict Management[J]. Applied Mathematics and Computation. 83,117-152.1997.

[11] Li KW, Hipel KW, Kilgour DM. Fang L Preference uncertainty in the graph model for conflict resolution[J]. IEEE Trans Syst Man CybernPart A. Syst Hum, 2004, 3(4): 507-520. 